\title{
Properties of the Variation of the Infrared Emission of OH/IR Stars III. The $M$ Band Light Curves
}

\author{
Young-Joo Kwon and Kyung-Won Suh ${ }^{\dagger}$ \\ Department of Astronomy and Space Science, Chungbuk National University, Cheongju 361-763, Korea
}

To study properties of the pulsation in the infrared emission for long period variables, we have collected and analyzed the infrared observational data at $M$ band for $12 \mathrm{OH} / \mathrm{IR}$ stars. We present the light curves using the data that cover about 30 years including recent observations of ISO and Spitzer. We use Marquardt-Levenberg algorithm to determine the pulsation periods and amplitudes and compare them with previous results of infrared and radio investigations. Generally, the newly determined pulsation parameters show much less errors because of the larger database. We find that the relationship between the pulsation period and amplitudes at $M$ band is fairly well fitted with a simple linear equation in a wide period range. For $\mathrm{OH}$ 42.3-0.1, we find some evidences that the object could be a post-asymptotic giant branch star.

Keywords: stars: asymptotic giant branch and post-asymptotic giant branch, infrared: stars, stars: oscillations, circumstellar matter, dust: extinction

\section{INTRODUCTION}

Stars with low and intermediate mass evolve into the asymptotic giant branch (AGB) before the evolutionary stage of planetary nebulae. The $\mathrm{OH} / \mathrm{IR}$ stars are generally believed to be the last evolutionary phase of an oxygenrich AGB star emitting $\mathrm{OH}$ maser at 1,612 $\mathrm{MHz}$. OH/IR stars are characterized by the Mira-type long-period variable stars (LPVs) with large amplitude (>2.5 mag at optical). Shock waves produced by the strong pulsation and radiation pressure on the newly formed dust grains drive dusty stellar winds of high mass-loss rates $\left(10^{-8}-10^{-4} \mathrm{M}_{\odot} /\right.$ yr). The enhanced mass loss by stellar pulsation involves the opaque circumstellar envelope.

The Infrared Space Observatory (ISO, Kessler et al. 1996) with improved sensitivity and angular resolution had performed photometric, spectroscopic and polarimetric observations. The two spectrometers (SWS and LWS), a camera, and an imaging photo-polarimeter (ISOPHOT) covered vast wavelength range of 2.5-240 $\mu \mathrm{m}$ with high spatial resolutions. Spitzer Space Telescope (Spitzer) has the sensitive infrared array camera (IRAC) designed for observing faint sources such as brown dwarfs and protoplanetary system with deep large-area (Fazio et al. 2004). Four channels of IRAC provide simultaneous broadband images with high sensitivity at 3.6, 4.5, 5.8 and $8.0 \mu \mathrm{m}$. IRAC photometry at 3.6 and $4.5 \mu \mathrm{m}$ bands are similar to classical ground-based photometric system at $L$ and $M$ band, respectively. Thanks to ISO and Spitzer observations, we could collect valuable data points for OH/IR stars.

Suh \& Kwon (2009, hereafter paper I) and Kwon \& Suh (2010, hereafter paper II) investigated the $K$ band and $L$ band light curves of OH/IR stars, respectively. In this paper, we study pulsation properties at $M$ band for selected $12 \mathrm{OH} / \mathrm{IR}$ stars. We make the $M$ band light curves and determine the pulsation parameters by analyzing the light curves. We discuss the physical meaning of the parameters and evolutionary properties of the OH/IR stars. (c) This is an Open Access article distributed under the terms of the Creative Commons Attribution Non-Commercial License (http://creativecommons.org/licenses/by-nc/3.0/) which permits unrestricted non-commercial use, distribution, and reproduction in any medium, provided the original work is properly cited.
Received Nov 15, 2010 Revised Nov 30, 2010 Accepted Dec 03, 2010 ${ }^{\dagger}$ Corresponding Author

E-mail: kwsuh@chungbuk.ac.kr

Tel: +82-43-261-2315 Fax: +82-43-274-2312 


\section{SAMPLE STARS}

For this paper, we choose $12 \mathrm{OH} / \mathrm{IR}$ stars which have a wide range of pulsation periods (400-1,800 days). The sample stars are same as those used in paper II. We present the $\mathrm{OH}$ name of sample stars, the general catalogue of variable stars (GCVS) name, the galactic position and the distance in Table 1. The galactic positions of stars are based on the SIMBAD database. The distances of $\mathrm{OH}$ 45.5+0.1 and 32.8-0.3 refer to Nyman et al. (1993), the distances of the others are from Lepine et al. (1995).

The detailed spectral observations by ISO provide useful data. The short wavelength spectrometer (SWS) ob-

Table 1. Sample stars.

\begin{tabular}{clccc}
\hline OH name & GCVS & $\begin{array}{c}\text { l. } \\
\text { (degree) }\end{array}$ & $\begin{array}{c}\text { b. } \\
\text { (degree) }\end{array}$ & $\begin{array}{c}\text { Dist. } \\
\text { (kpc) }\end{array}$ \\
\hline $26.2-0.6$ & V0438 Sct & 26.2092 & -00.5893 & 4.1 \\
$26.4-1.9$ & V0440 Sct & 26.4221 & -01.9346 & 3.4 \\
$26.5+0.6$ & V0437 Sct & 26.5434 & +00.6178 & 1.4 \\
$28.7-0.6$ & V0439 Sct & 28.7192 & -00.5787 & 2.8 \\
$30.1-0.2$ & V1361 Aql & 30.1369 & -00.2322 & 3.3 \\
$30.7+0.4$ & V1360 Aql & 30.7171 & +00.4278 & 5.2 \\
$32.8-0.3$ & V1365 Aql & 32.8271 & -00.3155 & 3.9 \\
$39.9+0.0$ & V1367 Aql & 39.9162 & +00.0182 & 5.6 \\
$42.3-0.1$ & V1368 Aql & 42.3081 & -00.1330 & 6.5 \\
$45.5+0.1$ & V1369 Aql & 45.4722 & +00.0749 & 2.5 \\
$127.8+0.0$ & V669 Cas & 127.8147 & -00.0208 & 1.6 \\
$178.0-31.4$ & IKTau & 177.9544 & -31.4119 & 0.3 \\
\hline
\end{tabular}

GCVS: general catalogue of variable stars. served medium and high spectral resolution in the wide wavelength region of 2.38-45.2 $\mathrm{m}$ (de Graauw et al. 1996). ISOPHOT (PHT; Lemke et al. 1996) has four principal modes of detector element aperture photometry (3$120 \mu \mathrm{m})$, array imaging (40-240 $\mu \mathrm{m})$, polarimetry (25-170 $\mu \mathrm{m})$ and spectrophotometry (2.5-12 $\mu \mathrm{m})$. In this work, we use spectral observation data of SWS and PHT for flux density of $M$ band at $4.8 \mu \mathrm{m}$.

The Galactic Legacy Infrared Mid-Plane Survey Extraordinaire (GLIMPSE, Benjamin et al. 2003), one of the Spitzer Legacy Science Program, observed our galactic plane with the IRAC on board the Spitzer. We use the observation data of IRAC at $4.5 \mu \mathrm{m}$ band which shares similarity with the standard $M$ band.

All the sample stars except IK Tau and $\mathrm{OH} 127.8+0.0$ were observed by GLIMPSE survey, but all the data of observed stars are contaminated by electronic artifacts such as saturation, muxbleed, and column pull-down. The sensitivity of IRAC is too high to observe the bright $\mathrm{OH} / \mathrm{IR}$ stars. According to GLIMPSE3D v1.0 data release document, the saturation limit for GLIMPSE is 6.5 mag at $4.5 \mu \mathrm{m}$ band.

The photometry for some softly saturated stars can be extracted using the wings surrounding a saturated source (see paper II). We use the corrected flux density of the some softly saturated stars from the GLIMPSE source catalogs. The Spitzer data of the highly saturated sources (marked by parenthesis in Table 2) are not used for our analysis, but presented in the light curves as lower limits.

Table 2 lists the number of observational data used

Table 2. The $M$ band observations for the sample $\mathrm{OH} / \mathrm{IR}$ stars.

\begin{tabular}{|c|c|c|c|c|c|c|c|c|c|c|c|c|}
\hline References & 26.2-0.6 & 26.4-1.9 & $26.5+0.6$ & 28.7-0.6 & $30.1-0.2$ & $30.7+0.4$ & $32.8-0.3$ & $39.9+0.0$ & 42.3-0.1 & $45.5+0.1$ & $127.8+0.0$ & 178.0-31.4 \\
\hline Strecker \& Ney (1974) & & & & & & & & & & & & 3 \\
\hline Lebofsky et al. (1976) & & & & & & & & & & & 1 & \\
\hline Lebofsky et al. (1978) & & & 1 & & & & & & & & & \\
\hline Evans \& Beckwith (1977) & & & 3 & & 3 & & 2 & & & 4 & & \\
\hline Ney \& Merrill (1980) & & & 4 & & & & & & & & 1 & 13 \\
\hline Werner et al. (1980) & & & 1 & & 1 & & 1 & & & 1 & & \\
\hline Engels (1982) & 10 & $10(1)$ & $10(1)$ & 9 & 10 & 10 & 10 & 10 & 7 & 9 & & \\
\hline Willems \& de Jong (1982) & & 1 & 2 & & & & & & & & & \\
\hline Gullixson et al. (1983) & & & 1 & & & & & & & & 2 & \\
\hline Fix \& Mutel (1984) & & 1 & & & & & & & $1(1)$ & & & \\
\hline Herman et al. (1984) & 1 & 1 & & 1 & 1 & 1 & 1 & 1 & 1 & & & \\
\hline Gehrz et al. (1985) & 2 & $4(1)$ & & 3 & & 5 & & 3 & $2(1)$ & 1 & 3 & \\
\hline Persi et al. (1990) & & & & & & & & & & & 2 & \\
\hline Jones et al. (1990) & & & $11(1)$ & & & & & & & & 10 & 10 \\
\hline Fouque et al. (1992) & & & & & & & & & & & & 1 \\
\hline Nyman et al. (1993) & 1 & 1 & 1 & 1 & 1 & 1 & 1 & $1(1)$ & 1 & 1 & & \\
\hline Le Bertre (1993) & & & 15 & & & & & & & & & 15 \\
\hline Lepine et al. (1995) & & & 1 & & & & & & & & & \\
\hline ISO & 1 & & 1 & & & & 1 & & & & 2 & \\
\hline SPITZER & $1(1)$ & & & & & & & 1 & 1 & $1(1)$ & & \\
\hline Total number & $16(1)$ & $18(2)$ & $51(2)$ & 14 & 16 & 17 & 16 & $16(1)$ & $13(2)$ & $17(1)$ & 21 & 42 \\
\hline
\end{tabular}


for this paper. The numbers marked by parenthesis in Table 2 mean unused observation data for the analysis to determine the pulsation parameters (see Section 3). Compared with previous investigations, we use much larger database of recent observations including ISO and Spitzer. For example, we use 51 data points for $\mathrm{OH}$ $26.5+0.65$ obtained for about 22 years; the database is 5 times larger than that of Engels et al. (1983). The new pulsation parameters based on the larger database would show smaller errors.

To obtain the standard flux in $\mathrm{W} / \mathrm{m}^{2}$ for all the data, we use the zero-magnitude calibrating methods. The zeromagnitude calibrating data are taken from the related references.

\section{RESULTS AND DISCUSSION}

In Fig. 1, we plot the light curves for $12 \mathrm{OH} / \mathrm{IR}$ stars using the observation data (presented by symbols) at $M$ band with the best fitting sinusoidal curve (presented by a solid line). The sinusoidal curve, which is a function of time (t), is in the form

$$
\mathrm{y}_{0}+\mathrm{A} \sin (2 \pi \mathrm{t} / \mathrm{P}+\mathrm{q}),
$$

where $\mathrm{y}_{0}$ is the mean-brightness, $\mathrm{A}$ and $\mathrm{P}$ are the pulsation amplitude and period, and $\mathrm{q}$ is the phase parameter. For curve-fitting of the observational data, we use the Marquardt-Levenberg algorithm to find the coefficients of the individual variables (Press et al. 1986). We perform the curve-fitting using the same weighted value for all the data. By analyzing the light curves, we determine the new pulsation parameters: the period, amplitude, and mean brightness.

We neglect the long-term trends caused by a secondary period within the time range of observation data. The data points that do not follow the general trend are not used for determination of the pulsation parameters. These unused data points are marked by parenthesis in Table 2 and the light curves (Fig. 1).

In Table 3, we list the new pulsation parameters (period, amplitude, and mean brightness) obtained in this paper and previous investigations. The periods and amplitudes except marked ones in the column of previous works are taken from Engels et al. (1983) and the periods in radio band are from the $\mathrm{OH}$ maser observations by Herman \& Habing (1985). The period of IK Tau from Hale et al. (1997) was based on variations in $11 \mu \mathrm{m}$ flux.

Generally, the new pulsation parameters obtained in this paper show much smaller standard deviation errors than previous works because of the larger database. Only for $\mathrm{OH} 30.7+0.4$, our results show larger errors possibly due to unusual scatters in the new five data points of the object.

\subsection{Period and amplitude ( $\mathrm{P}-\mathrm{A})$ relation}

Fig. 2 shows the period-amplitude relation at $M$ band for $12 \mathrm{OH} / \mathrm{IR}$ stars obtained in this paper and previous investigations of Engels et al. (1983) and Le Bertre (1993). We find a general relationship between the period and amplitude for Mira-type LPVs at $M$ band. A linear relationship from 36 data points at $M$ band is Amp (M) $=2.81$ $\log$ (P_M)-6.57. The unused data points because of the too much scatter from the general trend are marked by parenthesis.

Table 3. New pulsation parameters.

\begin{tabular}{|c|c|c|c|c|c|c|}
\hline \multirow{2}{*}{ Objects } & \multicolumn{3}{|c|}{ Period (day) } & \multicolumn{2}{|c|}{ Amplitude (mag) } & \multirow[t]{2}{*}{$<\mathrm{m}>$ (mag) } \\
\hline & This work & Previous & Radio & This work & Previous & \\
\hline $26.2-0.6$ & $1,193 \pm 9.8$ & $1330 \pm 50$ & $1,181 \pm 13$ & $1.80 \pm 0.22$ & 1.77 & $2.57 \pm 0.18$ \\
\hline 26.4-1.9 & $493 \pm 4.8$ & $540 \pm 20$ & $652 \pm 26$ & $1.45 \pm 0.17$ & 1.37 & $2.18 \pm 0.14$ \\
\hline $26.5+0.6$ & $1,564 \pm 9.6$ & $1,630 \pm 100$ & $1,566 \pm 16$ & $2.25 \pm 0.13$ & 1.97 & $-0.36 \pm 0.09$ \\
\hline $28.7-0.6$ & $641 \pm 6.3$ & $640 \pm 10$ & $627 \pm 17$ & $2.00 \pm 0.23$ & 1.30 & $1.67 \pm 0.18$ \\
\hline $30.1-0.2$ & $923 \pm 8.5$ & $970 \pm 40$ & $853 \pm 21$ & $1.55 \pm 0.14$ & 1.52 & $2.10 \pm 0.10$ \\
\hline $30.7+0.4$ & $1,158 \pm 54.2$ & $1,140 \pm 30$ & $1,039 \pm 27$ & $2.55 \pm 0.27$ & 1.61 & $2.80 \pm 0.20$ \\
\hline $32.8-0.3$ & $1,729 \pm 15.5$ & $1,750 \pm 130$ & $1,536 \pm 10$ & $2.90 \pm 0.20$ & 2.68 & $2.70 \pm 0.15$ \\
\hline $39.9+0.0$ & $771 \pm 7.1$ & $770 \pm 20$ & $823 \pm 45$ & $2.00 \pm 0.27$ & 1.49 & $3.02 \pm 0.19$ \\
\hline $42.3-0.1$ & $1,723 \pm 24.9$ & $1,650 \pm 150$ & $1,945 \pm 83$ & $1.05 \pm 0.17$ & 0.72 & $4.44 \pm 0.13$ \\
\hline $45.5+0.1$ & $721 \pm 5.8$ & $720 \pm 20$ & $760 \pm 31$ & $1.50 \pm 0.19$ & 1.45 & $3.12 \pm 0.13$ \\
\hline $127.8+0.0$ & $1,506 \pm 13.8$ & $1,537 \pm 18^{\text {a) }}$ & $1,994 \pm 130$ & $2.00 \pm 0.18$ & $2.00^{\mathrm{a})}$ & $0.74 \pm 0.30$ \\
\hline IK Tau & $466 \pm 0.8$ & $470 \pm 7^{\mathrm{b})}$ & $455.6 \pm 57$ & $1.15 \pm 0.08$ & - & $-2.70 \pm 0.05$ \\
\hline
\end{tabular}

a) Suh \& Kim (2002), b) Hale et al. (1997). 

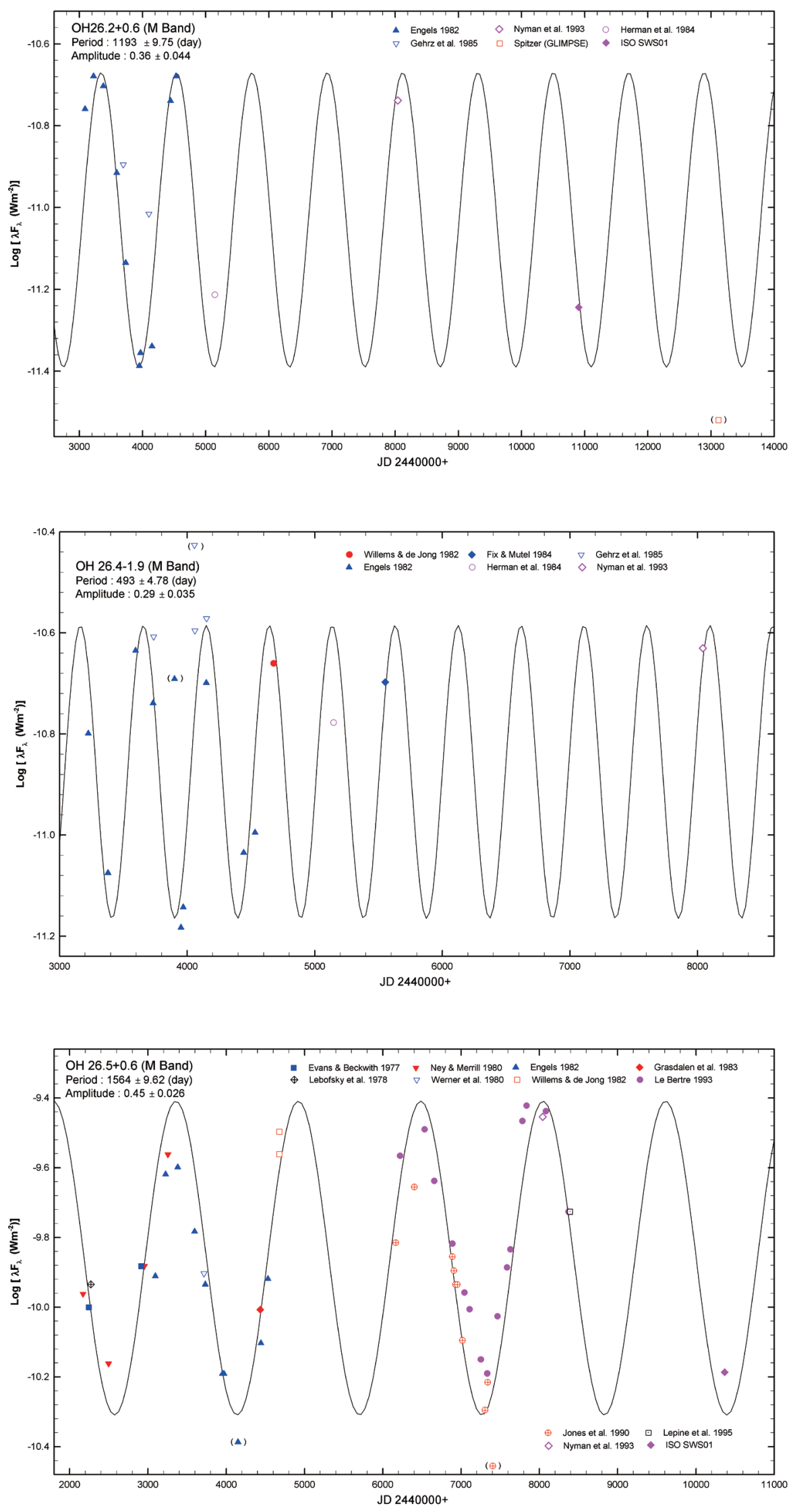

Fig. 1. $M$ band light curves of $\mathrm{OH} / \mathrm{IR}$ stars. 

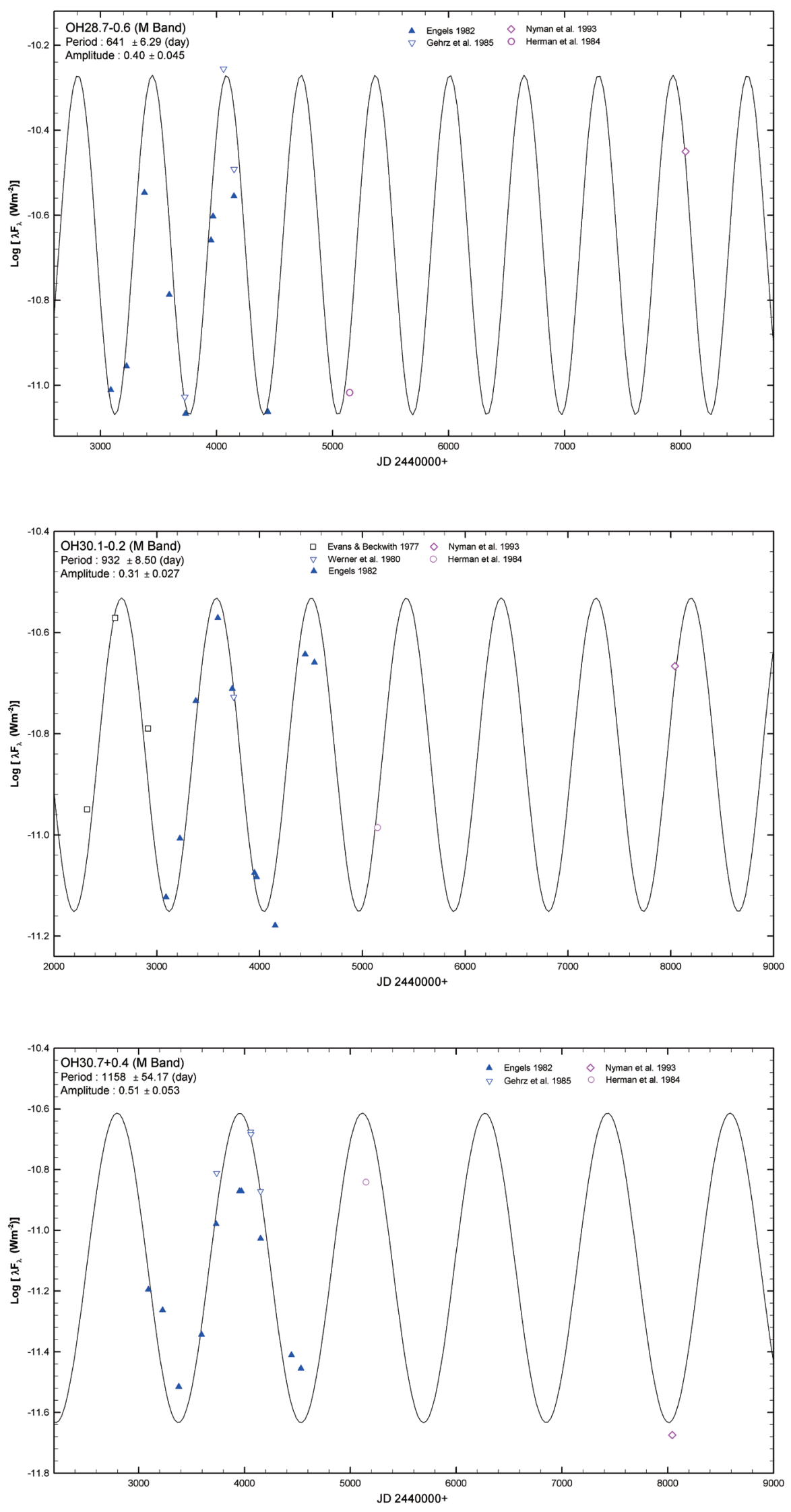

Fig. 1. (continued) 

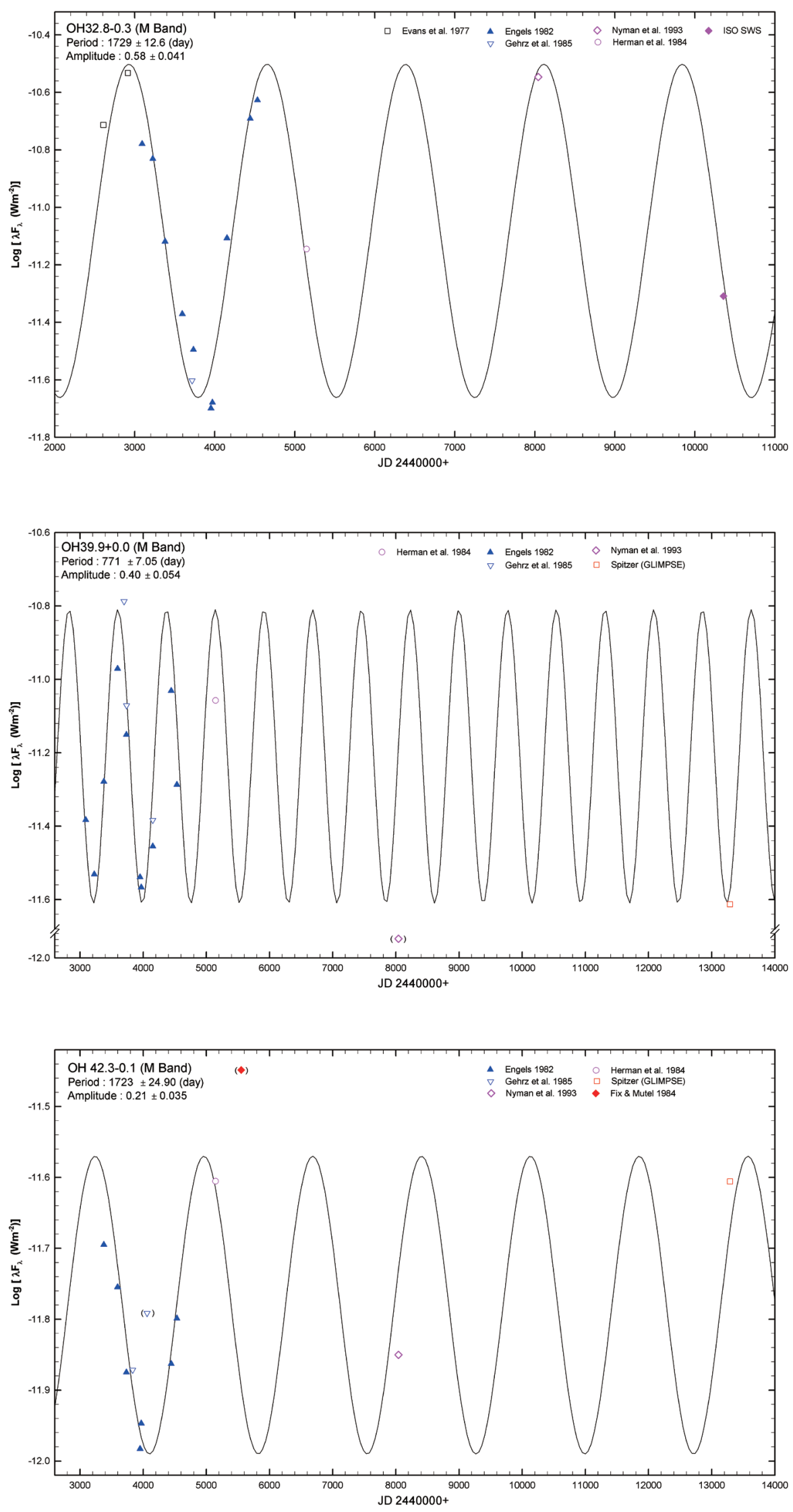

Fig. 1. (continued) 

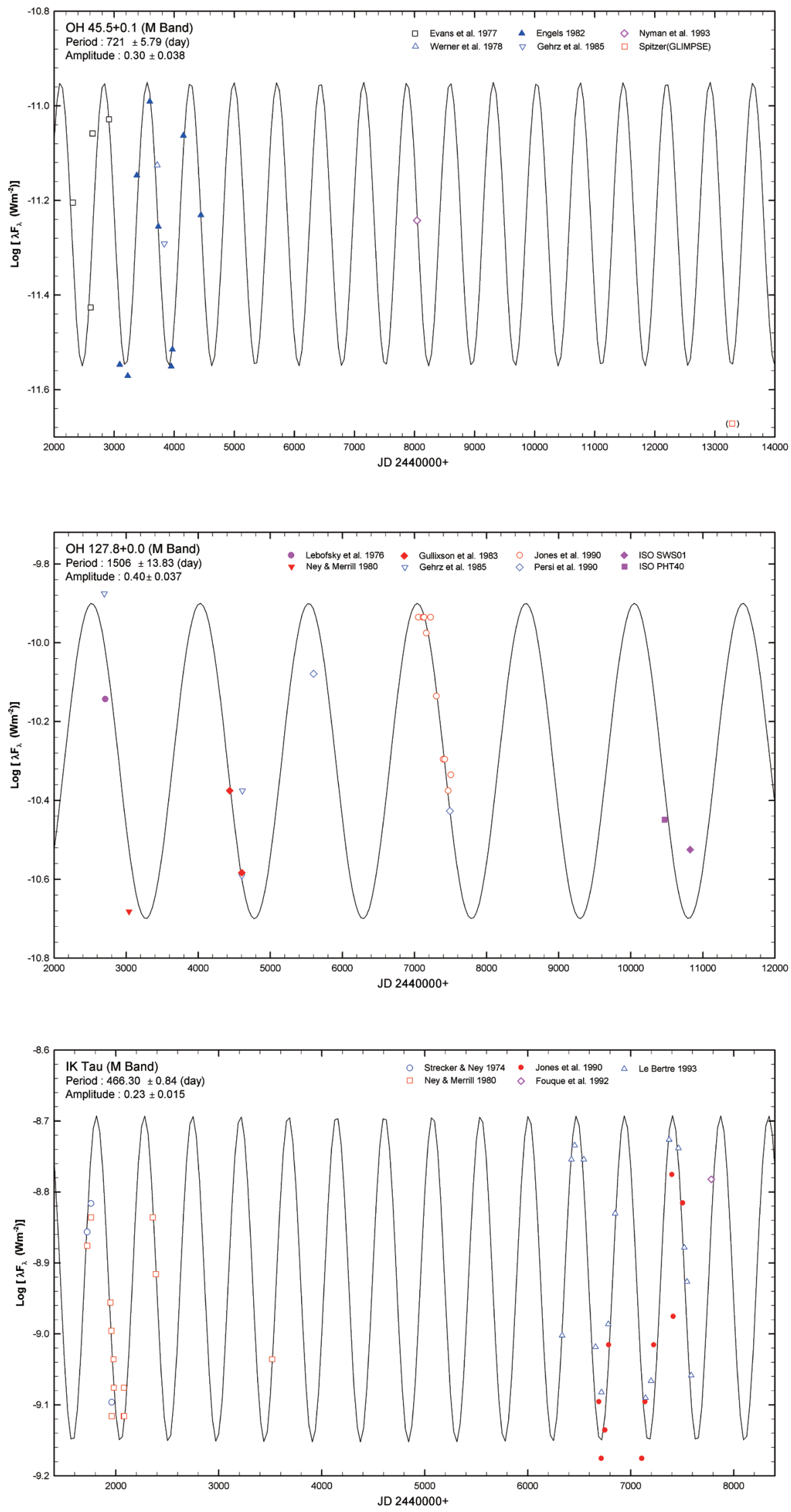

Fig. 1. (continued) 


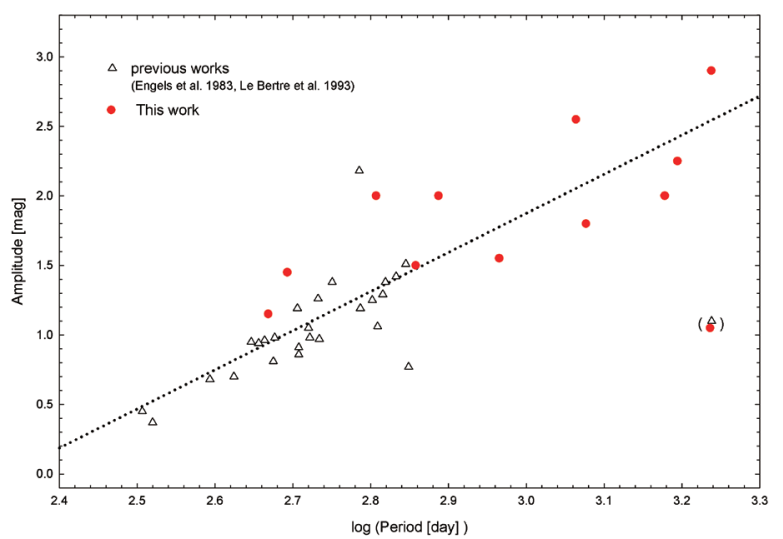

Fig. 2. P-A relation at $M$ band.

Table 4. P-A parameters at different bands.

\begin{tabular}{cccc}
\hline Band & Slope & Zero-point & Sample \# \\
\hline$K$ & $3.17 \pm 0.57$ & $-6.89 \pm 1.69$ & 17 \\
$L$ & $3.24 \pm 0.22$ & $-7.78 \pm 0.62$ & 55 \\
$M$ & $2.81 \pm 0.22$ & $-6.57 \pm 0.62$ & 36 \\
\hline
\end{tabular}

When we compare the P-A relation at three different bands, the $M$ band shows a less steep slope and lower zero-point than those of $K$ and $L$ band (see paper I and II). Table 4 lists the parameters for the P-A relation at three different bands. The slope of the P-A relation in the $L$ band is the steepest. Contrary to paper I, we cannot find different trends in the short and long period regions. This phenomenon could be due to the absorption effect (water and $\mathrm{CO}$ molecules) at $\mathrm{K}$ band only for low massloss rate O-rich AGB stars. However, the dust emission for the OH/IR star with high mass-loss rate fills the molecular absorption line. Furthermore, contribution of OH/IR stars with low mass-loss rate in Fig. 2 is much less than $\mathrm{P}-\mathrm{A}$ relation at $K$ band for selective effect.

\subsection{OH/IR stars with small amplitude pulsation}

In Fig. 2, there are two peculiar stars $(\mathrm{OH} 30.1-0.7$ and $\mathrm{OH}$ 42.3-0.1; marked by parenthesis in lower right corner) that have small pulsation amplitude for their long periods. The pulsation period and amplitude of $\mathrm{OH}$ 30.10.7 were determined by Engels et al. (1983). One of the reasonable explanations for this could be that the stars are on the transition phase from AGB to Proto-planetary nebula (post-AGB) after a super-wind phase. Mass-loss of the post-AGB stars during this time may not stop abruptly, but decrease steadily for $10^{2} \sim 10^{4}$ years (Bedijn 1987). Flux density of $\mathrm{SiO}$ and $\mathrm{H}_{2} \mathrm{O}$ maser emission in inner circumstellar envelope can be dwindled down as de- creasing of mass-loss rate.

Engels (2002) classified the $\mathrm{H}_{2} \mathrm{O}$ maser feature of young proto-planetary nebulae (PPN) into two types: type $\mathrm{A}$ and type B. Type A is AGB stars with low mass-loss rate $\left(<10^{-6}\right.$ $\mathrm{M} / \mathrm{yr}$ ) and the peak velocity is located close to the radial velocity of the stars. Stars of type B have higher mass loss rate and consist of two line complexes close to the velocities of the $\mathrm{OH}$ maser peaks.

For $\mathrm{OH}$ 42.3-0.1, water maser emission at $22 \mathrm{GHz}$ was observed several times. Olnon et al. (1980) observed that the $\mathrm{H}_{2} \mathrm{O}$ maser feature lies outside the $\mathrm{OH} 1,612 \mathrm{MHz}$ emission range toward this star. Gomez et al. (1994) suggested that the star is a high-velocity outflow source in the early stages of post-AGB phase because the velocity width of the $\mathrm{H}_{2} \mathrm{O}$ maser emission is larger than that of the $\mathrm{OH}$ maser emission. However, a recent observation of $\mathrm{H}_{2} \mathrm{O}$ emission feature by Kim et al. (2010) found that the peak velocity is located near the radial velocity of the central star.

For $\mathrm{OH}$ 42.3-0.1, we find evidences for decreasing mass-loss rate. The mass-loss rate changes from a higher level $\left(2.7 \times 10^{-4} \mathrm{M}_{\odot} / \mathrm{yr}\right.$ at outer region obtained by Justtanont et al. 2006) to the level lower than $10^{-6} \mathrm{M}_{\odot} / \mathrm{yr}$ in inner region (Kim et al. 2010). A threshold mass loss rate for $\mathrm{H}_{2} \mathrm{O}$ maser is about $10^{-8}-10^{-7} \mathrm{M}_{\odot} / \mathrm{yr}$ (Engels 2002). Thus the mass-loss rate at inner region for $\mathrm{OH}$ 42.3-0.1 would be between $10^{-8}$ and $10^{-6} \mathrm{M}_{\odot} / \mathrm{yr}$ and keep decreasing rapidly. However, the line profile of $\mathrm{H}_{2} \mathrm{O}$ maser can vary in time. Therefore, we need to monitor this star for longer time to be able to make a firm conclusion.

SiO maser emission is rarely detected among PPN candidates (Nyman et al. 1998). Therefore, the detection of $\mathrm{SiO}$ maser emission from this star (Jewell et al. 1991, Kim et al. 2010) supports the idea that $\mathrm{OH} 42.3-0.1$ could be a post-AGB star just leaving from AGB phase. If the star is really in the transition phase, the flux density of $\mathrm{SiO} / \mathrm{H}_{2} \mathrm{O}$ maser will decrease for about several hundred years and evolve into a non-variable.

$\mathrm{OH}$ 30.1-0.7 seems to be a typical OH/IR star. $\mathrm{H}_{2} \mathrm{O}$ maser at $22 \mathrm{GHz}$ is seen as a single-peaked spectrum near the blue-shifted OH peaks at $85 \mathrm{~km} / \mathrm{s}$ (Cato et al. 1976, Valdettaro et al. 2001, Deacon et al. 2007) and SiO maser emission is also seen (Nyman et al. 1998). We could not find any evidences for decreasing mass-loss rate. More observations in infrared and radio regions are necessary for further study.

\subsection{Comparison with the $L$ band data}

In paper II, we showed that the pulsation periods at $L$ 


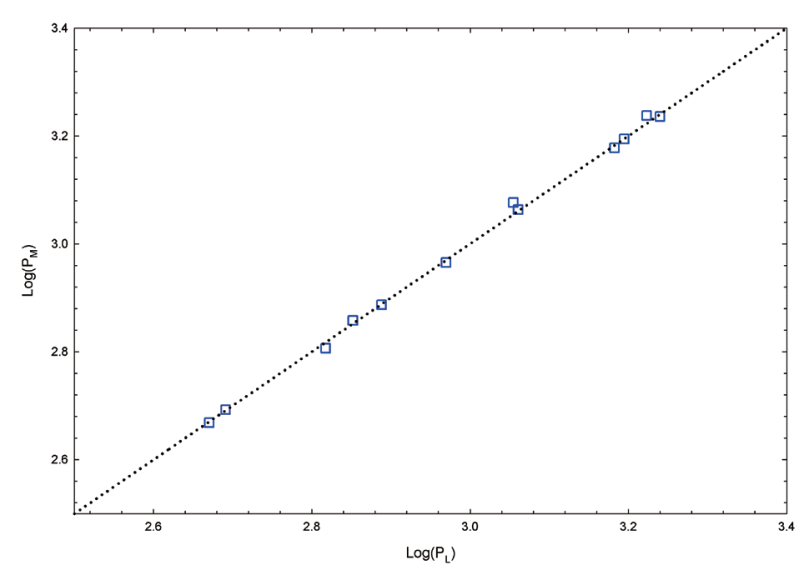

Fig. 3. Periods at $L$ and $M$ bands.

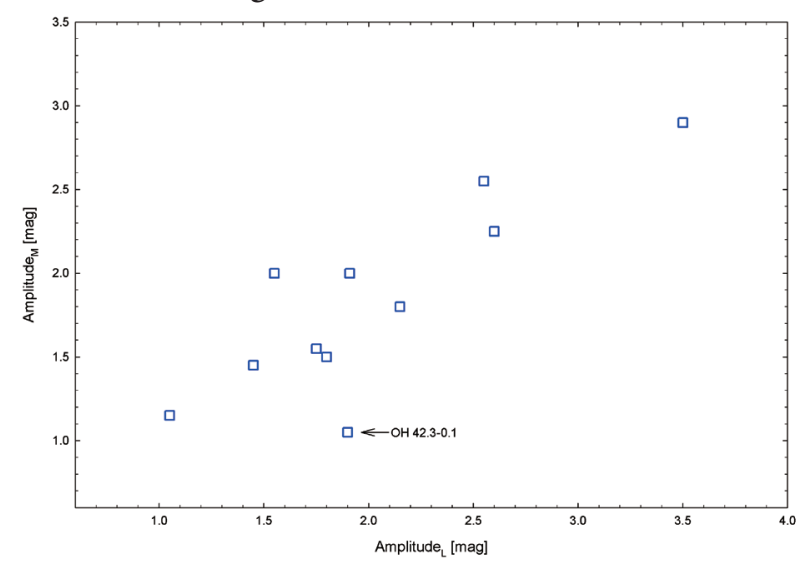

Fig. 4. Amplitudes at $L$ and $M$ bands.

and $K$ bands are similar for $9 \mathrm{OH} / \mathrm{IR}$ stars. In this paper, we find that the pulsation periods at $M$ and $L$ bands are very similar for $12 \mathrm{OH} / \mathrm{IR}$ stars (Fig. 3).

The amplitude relationship (Fig. 4) also shows a linear relation between $L$ and $M$ band, but more scatters are seen. As is general for pulsating stars, the amplitudes at shorter wavelength ( $L$ band) appear to be larger than those at longer one ( $M$ band). The position of $\mathrm{OH} 42.3$ 0.1 locates on the amplitude relation diagram quite lower. The star shows small amplitude pulsation at $L$ and $M$ bands as we discussed in last subsection.

\section{CONCLUSIONS}

We have made the light curves at $M$ band for $12 \mathrm{OH} /$ IR stars. We have determined the pulsation parameters of the sample stars and compared them with previous results of the infrared and radio investigations. Generally, the newly determined pulsation parameters show much less errors than previous investigations because of the larger database. We find a simple linear relationship be- tween the period and amplitude at $M$ band. Like $L$ band light curves (paper II), there is no difference in the trends for different period regions.

We find that $\mathrm{OH}$ 42.3-0.1 with small amplitude pulsation could be a post-AGB star because of some evidences in the maser emission properties. However, we could not find any evidence of a post-AGB star for $\mathrm{OH}$ 30.1-0.7 with small amplitude pulsation. Further investigations are necessary for these stars.

\section{ACKNOWLEDGEMENTS}

This work was supported by the Korea Science and Engineering Foundation (KOSEF) grant funded by the Korea government (MEST). This research has made use of the SIMBAD database, operated at CDS, Strasbourg, France and the NASA/ IPAC Infrared Science Archive, which is operated by the Jet Propulsion Laboratory, California Institute of Technology, under contract with the National Aeronautics and Space Administration.

\section{REFERENCES}

Bedijn, P. J. 1987, A\&A, 186, 136

Benjamin, R. A., Churchwell, E., Babler, B. L., Bania, T. M., Clemens, D. P., Cohen, M., Dickey, J. M., Indebetouw, R., Jackson, J. M., Kobulnicky, H. A., Lazarian, A., Marston, A. P., Mathis, J. S., Meade, M. R., Seager, S., Stolovy, S. R., Watson, C., Whitney, B. A., Wolff, M. J., \& Wolfire, M. G. 2003, PASP, 115, 953, doi: 10.1086/376696

Cato, B. T., Ronnang, B. O., Rydbeck, O. E. H., Lewin, P. T., Yngvesson, K. S., Cardiasmenos, A. G., \& Shanley, J. F. 1976, ApJ, 208, 87, doi: 10.1086/154584

Deacon, R. M., Chapman, J. M., Green, A. J., \& Sevenster, M. N. 2007, ApJ, 658, 1096 doi: 10.1086/511383

de Graauw, T., Haser, L. N., Beintema, D. A., Roelfsema, P. R., van Agthoven, H., Barl, L., Bauer, O. H., Bekenkamp, H. E. G., Boonstra, A. J., Boxhoorn, D. R., Cote, J., de Groene, P., van Dijkhuizen, C., Drapatz, S., Evers, J., Feuchtgruber, H., Frericks, M., Genzel, R., Haerendel, G., Heras, A. M., van der Hucht, K. A., van der Hulst, T., Huygen, R., Jacobs, H., Jakob, G., Kamperman, T., Katterloher, R. O., Kester, D. J. M., Kunze, D., Kussendrager, D., Lahuis, F., Lamers, H. J. G. L. M., Leech, K., van der Lei, S., van der Linden, R., Luinge, W., Lutz, D., Melzner, F., Morris, P. W., van Nguyen, D., Ploeger, G., Price, S., Salama, A., Schaeidt, S. G., Sijm, N., Smoorenburg, C., Spakman, J., Spoon, H., Steinmayer, M., Stoecker, J., Valentijn, E. A., 
Vandenbussche, B., Visser, H., Waelkens, C., Waters, L. B. F. M., Wensink, J., Wesselius, P. R., Wiezorrek, E., Wieprecht, E., Wijnbergen, J. J., Wildeman, K. J., \& Young, E. 1996, A\&A, 315, 49

Engels, D. 1982, Zur Natur von OH/IR-Objekten (Bonn: Duemmlers Verlag)

Engels, D. 2002, A\&A, 388, 252, doi: 10.1051/0004-6361: 20020483

Engels, D., Kreysa, E., Schultz, G. V., \& Sherwood, W. A. 1983, A\&A, 124, 123

Evans, N. J. II \& Beckwith, S. 1977, ApJ, 217, 729, doi: 10.1086/ 155619

Fazio, G. G., Hora, J. L., Allen, L. E., Ashby, M. L. N., Barmby, P., Deutsch, L. K., Huang, J. S., Kleiner, S., Marengo, M., Megeath, S. T., Melnick, G. J., Pahre, M. A., Patten, B. M., Polizotti, J., Smith, H. A., Taylor, R. S., Wang, Z., Willner, S. P., Hoffmann, W. F., Pipher, J. L., Forrest, W. J., McMurty, C. W., McCreight, C. R., McKelvey, M. E., McMurray, R. E., Koch, D. G., Moseley, S., Arendt, R. G., Mentzell, J. E., Marx, C. T., Losch, P., Mayman, P., Eichhorn, W., Krebs, D., Jhabvala, M., Gezari, D. Y., Fixsen, D. J., Flores, J., Shakoorzadeh, K., Jungo, R., Hakun, C., Workman, L., Karpati, G., Kichak, R., Whitley, R., Mann, S., Tollestrup, E. V., Eisenhardt, P., Stern, D., Gorjian, V., Bhattacharya, B., Carey, S., Nelson, B. O., Glaccum, W. J., Lacy, M., Lowrance, P. J., Laine, S., Reach, W. T., Stauffer, J. A., Surace, J. A., Wilson, G., Wright, E. L., Hoffman, A., Domingo, G., \& Cohen, M. 2004, ApJS, 154, 10, doi: 10.1086/422843

Fix, J. D. \& Mutel, R. L. 1984, AJ, 89, 406, doi: 10.1086/113528 Fouque, P., Le Bertre, T., Epchtein, N., Guglielmo, F., \& Kerschbaum, F. 1992, A\&AS, 93, 151

Gehrz, R. D., Hackwell, J. A., Grasdalen, G. L., Kleinmann, S. G., \& Mason, S. 1985, ApJ, 290, 296, doi: 10.1086/162985

Gomez, Y., Rodriguez, L. F., Contreras, M. E., \& Moran, J. M. 1994, RMxAA, 28, 97

Gullixson, C., Gehrz, R. D., Hackwell, J. A., Grasdalen, G. L., \& Castelaz, M. 1983, ApJS, 53, 413, doi: 10.1086/190897

Hale, D. D. S., Bester, M., Danchi, W. C., Hoss, S., Lipman, E., Monnier, J. D., Tuthill, P. G., Townes, C. H., Johnson, M., Lopez, B., \& Geballe, T. R. 1997, ApJ, 490, 407

Herman, J. \& Habing, H. J. 1985, A\&AS, 59, 523

Herman, J., Isaacman, R., Sargent, A., \& Habing, H. 1984, A\&A, 139, 171

Jewell, P. R., Snyder, L. E., Walmsley, C. M., Wilson, T. L., \& Gensheimer, P. D. 1991, A\&A, 242, 211

Jones, T. J., Bryja, C. O., Gehrz, R. D., Harrison, T. E., Johnson, J. J., Klebe, D. I., \& Lawrence, G. F. 1990, ApJS, 74, 785, doi: 10.1086/191518

Justtanont, K., Olofsson, G., Dijkstra, C., \& Meyer, A. W. 2006, A\&A, 450, 1051
Kessler, M. F., Steinz, J. A., Anderegg, M. E., Clavel, J., Drechsel, G., Estaria, P., Faelker, J., Riedinger, J. R., Robson, A., Taylor, B. G., \& Ximénez de Ferrán, S. 1996, A\&A, 315, 27

Kim, J. H., Cho, S. H., Oh, C. S., \& Byun, D. Y. 2010, ApJS, 188, 209, doi: 10.1088/0067-0049/188/1/209

Kwon, Y. J. \& Suh, K. W. 2010, JKAS, 43, 123 (paper II)

Le Bertre, T. 1993, A\&AS, 97, 729

Lebofsky, M. J., Kleinmann, S. G., Rieke, G. H., \& Low, F. J. 1976, ApJ, 206, 157, doi: 10.1086/182157

Lebofsky, M. J., Sargent, D. G., Kleinmann, S. G., \& Rieke, G. H. 1978, ApJ, 219, 487, doi: 10.1086/155804

Lemke, D., Klaas, U., Abolins, J., Abraham, P., Acosta-Pulido, J., Bogun, S., Castaneda, H., Cornwall, L., Drury, L., Gabriel, C., Garzon, F., Gemuend, H. P., Groezinger, U., Gruen, E., Haas, M., Hajduk, C., Hall, G., Heinrichsen, I., Herbstmeier, U., Hirth, G., Joseph, R., Kinkel, U., Kirches, S., Koempe, C., Kraetschmer, W., Kreysa, E., Krueger, H., Kunkel, M., Laureijs, R., Luetzow-Wentzky, P., Mattila, K., Mueller, T., Pacher, T., Pelz, G., Popow, E., Rasmussen, I., Rodriguez Espinosa, J., Richards, P., Russell, S., Schnopper, H., Schubert, J., Schulz, B., Telesco, C., Tilgner, C., Tuffs, R., Voelk, H., Walker, H., Wells, M., \& Wolf, J. 1996, A\&A, 315, 64

Lepine, J. R. D., Ortiz, R., \& Epchtein, N. 1995, A\&A, 299, 453 Ney, E. P. \& Merrill, K. M. 1980, Study of Sources in AFGL Rocket Infrared Study (Air Force Geophysical Laboratory) AFGL-TR-80-0050

Nyman, L. A., Hall, P. J., \& Le Bertre, T. 1993, A\&A, 280, 551

Nyman, L. A., Hall, P. J., \& Olofsson, H. 1998, A\&AS, 127, 185, doi: 10.1051/aas:1998343

Olnon, F. M., Winnberg, A., Matthews, H. E., \& Schultz, G. V. 1980, A\&AS, 42, 119

Persi, P., Ferrari-Toniolo, M., Ranieri, M., Marenzi, A., \& Shivanandan, K. 1990, A\&A, 237, 153

Press, W. H., Flannery, B. P., Teukolsy, S. A., \& Vetterling, W. T. 1986, Numerical Recipes (Cambridge: Cambridge Unversity Press)

Strecker, D. W. \& Ney, E. P. 1974, AJ, 79, 1410, doi: 10.1086/ 111693

Suh, K. W. \& Kim, H. Y. 2002, A\&A, 391, 665 doi: 10.1051/00046361:20020871

Suh, K. W. \& Kwon, Y. J. 2009, JASS, 26, 279 (paper I)

Valdettaro, R., Palla, F., Brand, J., Cesaroni, R., Comoretto, G., Di Franco, S., Felli, M., Natale, E., Palagi, F., Panella, D., \& Tofani, G. 2001, A\&A, 368, 845, doi: 10.1051/00046361:20000526

Werner, M. W., Beckwith, S., Gatley, I., Sellgren, K., Berriman, G., \& Whiting, D. L. 1980, ApJ, 239, 540, doi: $10.1086 / 158139$

Willems, F. \& de Jong, T. 1982, A\&A, 115, 213 